\title{
Clinical and Microbiological Profile of Malignant Otitis Externa in a Tertiary Care Center
}

\author{
Vishnu Vinayakumar1 ${ }^{1}$, Manoj Gopalan², Sathya Bhama ${ }^{3}$, Satheesh Somaraj ${ }^{4}$ \\ 1, 2, 4 Department of Otorhinolaryngology, Government Medical College, Thiruvananthapuram, Kerala, India. \\ ${ }^{3}$ Department of Microbiology, Government Medical College, Thiruvananthapuram, Kerala, India.
}

\section{ABSTRACT}

\section{BACKGROUND}

Malignant Otitis Externa (MOE) is a vicious form of inflammation of external auditory meatus. This study was aimed at analysing the demographic, clinical status, microbiological status, comorbidities and management of MOE.

\section{METHODS}

This descriptive study of patients with MOE who underwent treatment at the ENT Department of Govt. Medical College, Thiruvananthapuram was conducted from November 2017 to September 2019.

\section{RESULTS}

Forty-six patients with malignant otitis externa were treated as inpatients at the ENT Department of our hospital during the period of study. Among them, 30 were males and 16 were females. All of them were diabetic. Nocturnal earache was the commonest symptom in all patients. Oedema and granulation in the bony cartilaginous junction of external auditory meatus were the most common signs noted. Facial nerve palsy was seen in nearly half of the patients. Half of these affected patients showed recovery. Pseudomonas aeruginosa was the most common organism observed in blood culture. Intravenous antibiotics were given for an average duration of one month in all these patients.

\section{CONCLUSIONS}

Malignant otitis externa presented with nocturnal earache and recalcitrant otitis externa. Diabetes mellitus was present in all patients. Pseudomonas aeruginosa was the most commonly seen organism in this condition. Fungal infection can also cause MOE; the most common organisms being Aspergillus and Candida. ESR and CRP showed reduction on treatment and may be used as a prognostic marker.

\section{KEY WORDS}

Malignant Otitis Externa, Skull Base Osteomyelitis, Pseudomonas aeruginosa, Diabetes Mellitus
Corresponding Author:

Dr. Manoj Gopalan,

Sreelakam, SNRRA-133, Sreenarayana Guru Road, Ulloor Medical College P.O, Trivandrum- 695011, Kerala, India.

E-mail: manoj.g.ent @gmail.com

DOI: $10.14260 /$ jemds/2020/738

How to Cite This Article:

Vinayakumar V, Gopalan M, Bhama S, et al. Clinical and microbiological profile of malignant otitis externa in a tertiary care center. I Evolution Med Dent Sci 2020;9(45):3356-3361, DOI: $10.14260 /$ jemds/2020/738

Submission 20-07-2020,

Peer Review 30-09-2020,

Acceptance 06-10-2020,

Published 09-11-2020.

Copyright (C) 2020 Vishnu Vinayakumar et al. This is an open access article distributed under Creative Commons Attribution License [Attribution 4.0 International (CC $B Y 4.0)]$ 


\section{BACKGROUND}

Malignant otitis externa is a vicious infection of external auditory meatus in which the soft tissue pathogens have spread to the periosteum and temporal bone of the skull causing necrosis with or without progression to other bones of skull base. It was first described by Meltzer and Kelemen in 1959 as 'pyocutaneous osteomyelitis of the temporal bone'.1 Chandler named it malignant external otitis in 1968 when presenting his series of 13 patients with progressive osteomyelitis of the temporal bone. ${ }^{2}$ 'Necrotizing otitis externa' term may be used for aggressive soft tissue infection without bony involvement. ${ }^{3}$ Otogenic 'skull base osteomyelitis' refers to the more severe clinical entity following spread of infection through the soft tissue and cartilage with histological or radiological confirmed bony involvement. This aggressive infection commonly occurs in people with decreased immunity, such as those with diabetes mellitus, AIDS, chemotherapy induced pancytopenia, refractory anaemia, renal transplantation, chronic leukaemia, lymphoma and splenectomy. 4 Proper identification of predisposing factors and pathogenic agents are important for the success of the therapy and to prevent recurrence. Treatment of this disease is primarily medical. The antibiotics are decided according to the pus culture and sensitivity results. Surgery has no role in the treatment of MOE. Most of the time, surgery is limited to biopsy of granulation tissue. This study aims to describe the clinical features, predisposing factors, microbiological profile and outcome of clinically diagnosed malignant otitis externa cases.

\section{METHODS}

This descriptive study consists of all malignant otitis externa cases admitted in the Department of ENT, Government medical college, Thiruvananthapuram from November 2017 to September 2019. This study was approved by the Ethics committee, Trivandrum medical college. 46 patients clinically diagnosed as malignant otitis externa were taken up for the study.

The minimum sample size was obtained as 36 on applying the formula $4 \mathrm{pq} / \mathrm{d}^{2}$ where $\mathrm{P}$ is the prevalence, $\mathrm{q}=100-\mathrm{p}$ and $\mathrm{d}$ is $20 \%$ of $\mathrm{p}(\mathrm{p}=73) .73 \%$ of subjects had Pseudomonas aeruginosa as the causative organism according to study by Vadisha Bhat et al. ${ }^{5}$ The diagnosis of MOE was made according to the Levenson's criteria. It includes: 1 . Refractory otitis externa. 2. Severe nocturnal ear ache. 3. Purulent ear discharge 4. Granulation tissue in external canal 5. Presence of diabetes mellitus / other immunocompromised states. 6. Growth of Pseudomonas aeruginosa in specimen cultured from external canal. However, patients who were culture positive for other microorganisms were also included in this study. After obtaining written informed consent, details collected on a structured proforma was used to elicit the required information. Age, sex, predisposing risk factors, comorbidities, complications, duration of hospital stay, clinical and radiological features of the respondents were recorded along with the results of pus culture and sensitivity and histopathological examination.
Staging was done according to the clinical staging proposed by Thakur et al. ${ }^{4}$ Stage I - necrotizing external otitis, stage II - limited skull base osteomyelitis (lateral to jugular foramen) and stage III - Extensive skull base osteomyelitis (Jugular foramen and medial to it). Patients were grouped into 3 groups according to their $\mathrm{HbA} 1 \mathrm{c}$ results. ${ }^{6}$

\begin{tabular}{|cc|}
\hline HbA1c & Glycaemic Control \\
$>10$ & Very Poor \\
$7-10$ & Poor \\
$<7$ & Adequate \\
\hline Grouping of Patients According to HbA1c Levels \\
\hline
\end{tabular}

ESR and CRP were assessed at the time of admission and on follow-up after 2 months. Patients were grouped into 5 groups depending on their ESR at the time of admission and on follow-up.

\begin{tabular}{|cc|}
\hline ESR & Group \\
$<20$ & 1 \\
$20-39$ & 2 \\
$40-79$ & 3 \\
$80-100$ & 4 \\
$>100$ & 5 \\
\hline Grouping of Patients According to ESR \\
\hline
\end{tabular}

Patients were followed up for two months after discharge from hospital to assess the clinical outcome. Changes in the intensity of earache were scaled from 0 to 10 (no improvement to complete improvement based on the Wong Bakers pain faces scale-subjective measure).

In ear canal examination, presence of inflammation in the form of granulation / oedema or ear discharge was considered (objective measure). Patients were classified into 3 groups based on these measures.

\section{Statistical Analysis}

Statistical analysis was done with the help of SPSS version 16.0. Mean ESR before and after treatment was compared using paired $T$ test and mean CRP before and after treatment was compared using Wilcoxon signed rank test.

\section{RESULTS}

46 patients were admitted with malignant otitis externa in this study. Among them, 30 were males and 16 were females.

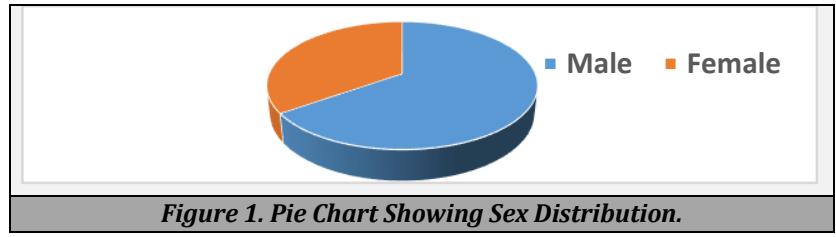

Age

60 - 70 age group was most commonly affected, the youngest being 45 years old and the oldest being 96 years old.

\section{Laterality}

Right ear MOE was seen in $54.34 \%$ of the patients and left ear MOE in $43.47 \%$. Bilateral MOE was very rare and was seen only in one patient. 


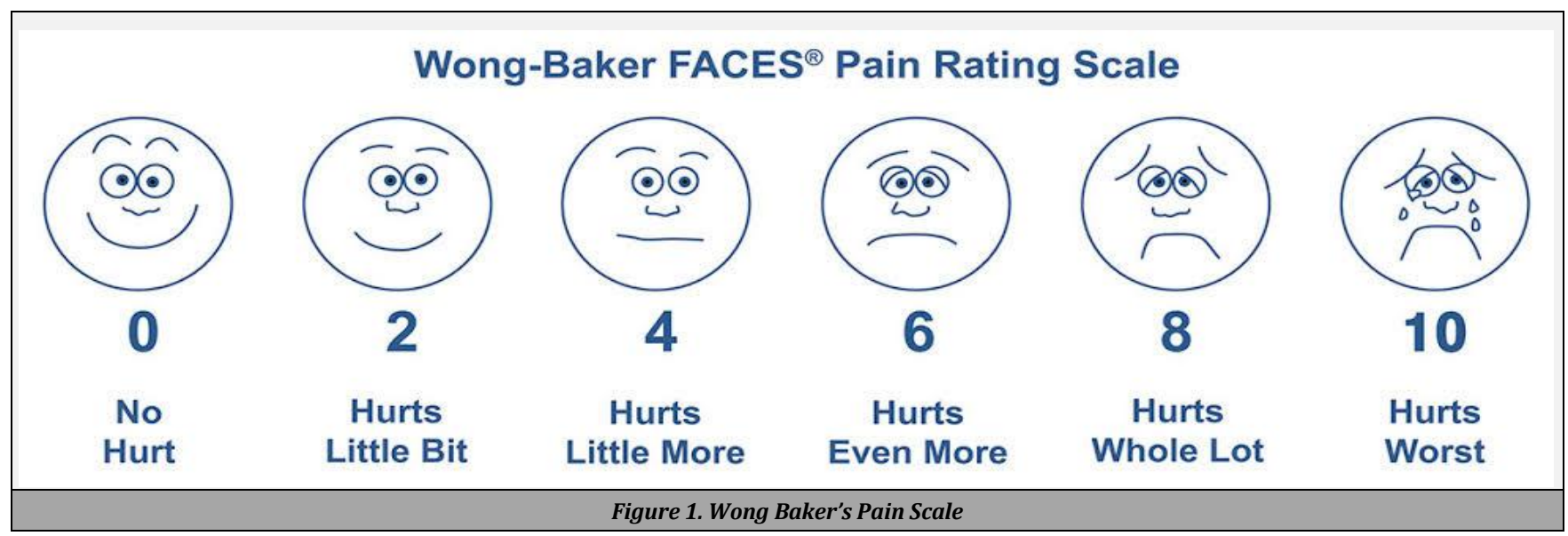

\section{Comorbidities}

All were diabetic. One patient was immunocompromised due to chemo radiation in addition. Most patients had associated hyperlipidaemia, hypertension and coronary artery disease. A few patients had chronic kidney disease and cerebrovascular disease as well. Right sided MOE was slightly more than left sided MOE.

\section{Clinical Features}

History of self-cleaning of ear with ear buds and other objects such as feather, match stick was present in most patients. A few patients gave history of water entry into ear and foreign body in the ear. All patients presented with characteristic nocturnal otalgia, purulent ear discharge and hard of hearing. $65.22 \%$ had associated conductive hearing loss, $26.1 \%$ had associated mixed hearing loss and $8.69 \%$ had associated sensorineural hearing loss. Granulation tissue was seen in all patients. Ear canal oedema and tragal tenderness were seen in $43.48 \%$ and $30.43 \%$ of the patients respectively. $13.04 \%$ of the patients had temporomandibular joint arthritis, $8.70 \%$ had associated parotitis and one patient had features of petrositis.

\section{Cranial Nerve Palsy}

19 patients (41.06\%) had facial nerve palsy of which 5 had grade IV facial nerve palsy, 10 had grade III facial nerve palsy and 4 patients had grade II facial nerve palsy. 3 patients had hypoglossal nerve palsy, 2 patients had glossopharyngeal and vagal nerve palsy, one had abducent nerve palsy and one had trigeminal nerve palsy in addition to facial nerve palsy.

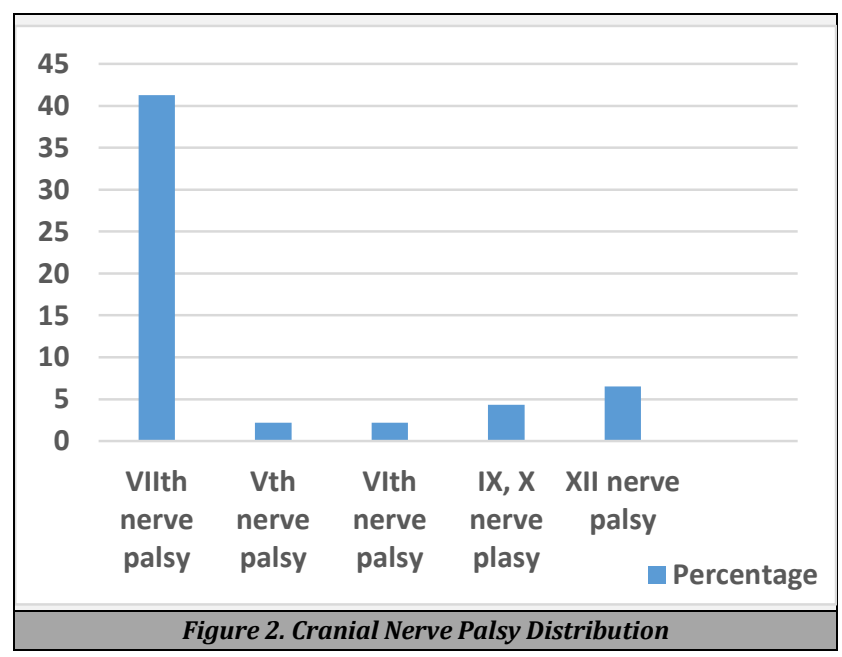

\section{Pus Culture and Sensitivity}

On doing pus culture and sensitivity, $80.44 \%$ of patients yielded positive culture results. Pseudomonas aeruginosa was the most common organism obtained (50\%) followed by Staphylococcus aureus (8.69\%), E. coli (4.35\%), Klebsiella (2.17\%) and Enterococcus (2.17\%). Fungal culture was positive in $13.04 \%$ of cases. There were 2 cases each of Aspergillus fumigatus and Candida albicans, one case each of Pseudallescheria boydii and Mucor. Culture was sterile for $19.56 \%$ of cases.

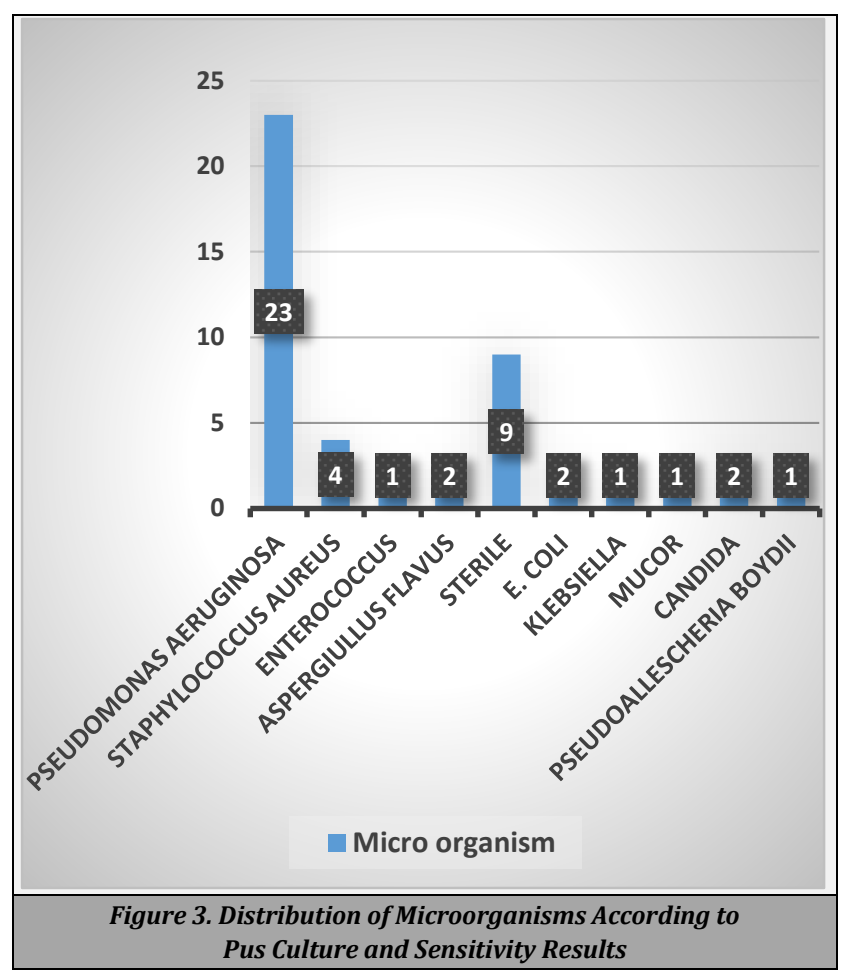

\section{Imaging}

HRCT (High-Resolution Computed Tomography) temporal bone was taken in all cases and MRI brain in a few cases with lower cranial nerve palsies. Soft tissue swelling in the external auditory meatus with its erosion was seen in all patients. Mastoid involvement was seen in $93.47 \%$ of the cases and TM (Temporo-Mandibular) joint involvement in $21.74 \%$ of the cases. Facial canal involvement was seen in $17.39 \%$ of the patients and stylomastoid involvement $23.91 \%$. $10.87 \%$ patients showed petrous apex extension, $19.57 \%$ showed 
nasopharyngeal extension and para pharyngeal extension was seen in $23.91 \%$ of the patients. $6.52 \%$ showed hypoglossal extension and $8.69 \%$ showed jugular fossa extension. One patient on doing MRI was found to have intracranial extension in the form of sigmoid sinus thrombophlebitis.

\section{Duration of Treatment}

$43.48 \%$ of the patient were treated for 4 - 6 weeks, $28.26 \%$ of the patients for 2 - 4 weeks, 19.7 of the patients for less than 2 weeks, 6.52 of the patients for 6 - 8 weeks and $2.17 \%$ of the patients for more than 2 months.

\section{Staging}

$54.35 \%$ of the patients were in stage I, $30.43 \%$ in stage II and $15.22 \%$ of the patients in stage III of malignant otitis externa.

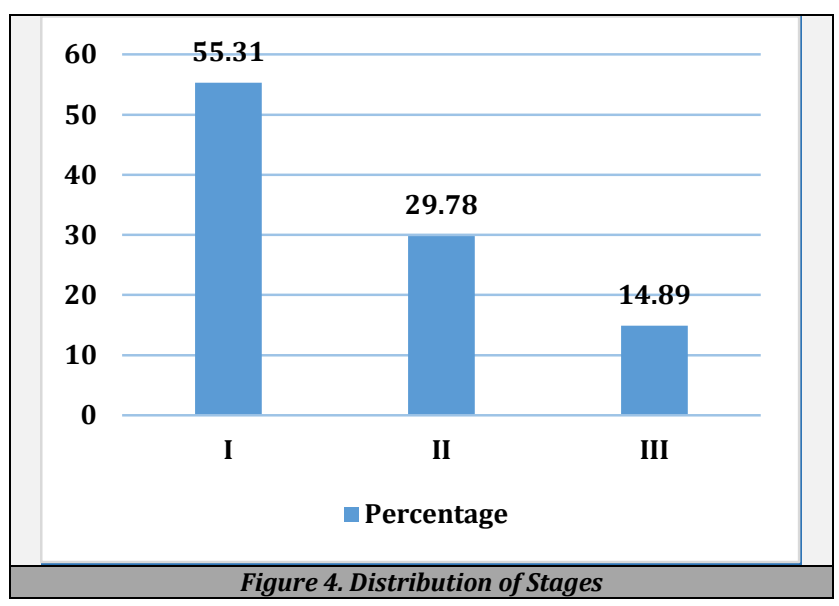

Patients were classified into 3 groups according to their glycaemic status.

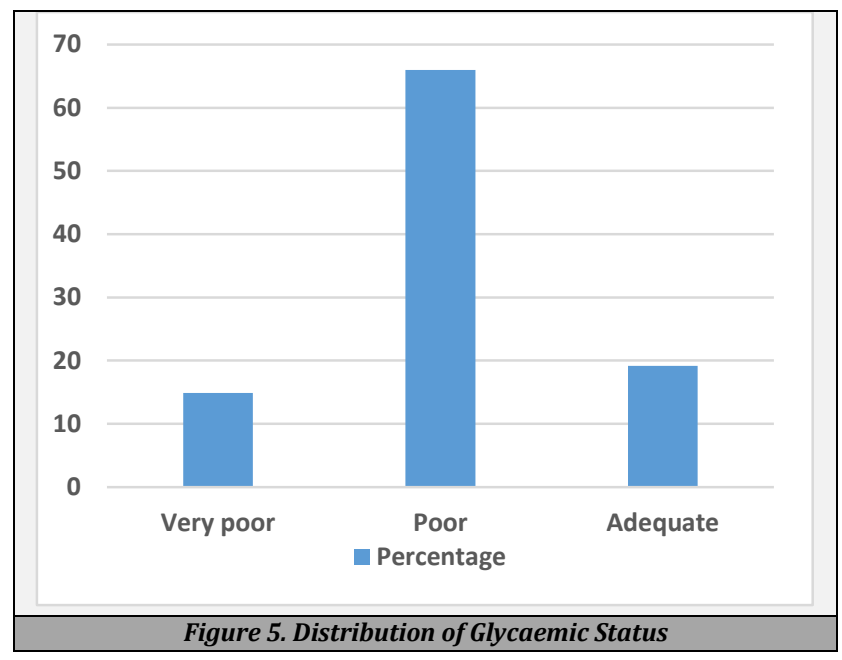

\section{Glycaemic Control}

$67.39 \%$ showed poor glycaemic control, $15.22 \%$ showed very poor glycaemic control whereas only $17.39 \%$ showed adequate glycaemic control.

\section{Treatment}

All patients were admitted and started on Inj. Ciprofloxacin. Antibiotic was later changed according to the results of pus culture and sensitivity. Biopsy of granulation tissue was sent for HPE (Histo-Pathological Examination) to rule out malignancy. Patients were grouped into 5 according to their ESR. Regular aural toileting and removal of granulation tissue was done. Patients' earache was graded according to Wong Baker's faces pain scale. Ear canal inflammation was also assessed depending on the presence of oedema / granulation tissue. Most of the patients had ESR more than 100 at presentation while at follow-up most patients had ESR between 20 and 40. On doing appropriate statistical tests, it was found that there was a significant reduction in ESR and CRP after treatment. 5 patients showed complete recovery of facial palsy from grade III of House Brackmann grading to grade I. 7 patients showed improvement in facial palsy from grade III to grade II and one patient showed recovery of facial palsy from grade IV to grade II. One patient showed recovery from glossopharyngeal and vagal nerve palsy. Another patient showed complete recovery of hypoglossal palsy.

\section{DISCUSSION}

46 patients were admitted with the diagnosis of MOE during the study period. All 46 of them had type II diabetes mellitus. Most of the authors have observed that diabetic patients were more susceptible to this infection. ${ }^{7,8}$ Diabetic patients were more affected because of endarteritis, microangiopathy, and small vessel obliteration caused by the disease. ${ }^{7}$

Patients having MOE presented with severe earache, purulent ear discharge, ear fullness and hard of hearing. Earache was very severe and out of proportion to findings and did not resolve after the usual treatment of otitis externa. Temporomandibular joint pain, headache, hemifacial pain and trismus were also seen with anterior extension of the disease.

Facial nerve paralysis was observed as the presenting feature in some patients. ${ }^{7}$ Facial nerve was the most commonly involved due to its proximity to the stylomastoid foramen. Facial palsy may also be due to involvement of facial nerve canal. In this study, 19 out of 46 patients had facial palsy and 3 had facial with hypoglossal palsy, 2 had facial with IX and $\mathrm{X}$ palsy, one had facial with trigeminal palsy and one had facial with abducent palsy. Rajput et al, in their case series of 21 cases, reported eight patients with cranial nerve involvement, five with facial nerve involvement, two with facial and vagal involvement, and one with facial and trigeminal involvment. 8 In 1985, Corey et al reported facial palsy in $70 \%$ of the patients. ${ }^{9}$ Mani et al $^{10}$ from their work found that the prognosis of MOE with facial nerve involvement was not different from those without facial nerve involvement.

$P$. aeruginosa was the most rampant organism found in pus culture reports. ${ }^{8}$ Others such as Staphylococcus aureus, $S$. epidermidis, Proteus mirabilis, Klebsiella oxytoca, and P. cepacia have also been found in MOE pus culture. In this study, out of the 46 patients, 23 (50\%) had P. aeruginosa. Staphylococcus aureus was isolated in 4 patients, E. coli in 2 patients and Klebsiella and Enterococcus in one patient each.

Fungal otitis externa was thought of if a patient with symptoms and signs of MOE did not show response to the appropriate culture directed antibiotic treatment for 2 weeks. The bacterial culture was negative in them. ${ }^{11}$ Fungal MOE was seen more in AIDS patients than in diabetics. ${ }^{7}$ In our study, fungus was isolated in $13.04 \%$. Aspergillus and Candida were 
the most common fungi isolated. One case each of Mucor and Pseudallescheria boydii was also present.

HRCT temporal bone was taken in all patients. All patients showed soft tissue density in external ear with erosion of bony EAC (External Auditory Canal). Other features such as involvement of stylomastoid foramen, jugular foramen and hypoglossal canal also looked for. Bone erosions are detected better in CT scans. Mani et al through their study found that CT findings had no correlation with the clinical course of MOE.10 A technetium-99m bone scan is positive in all cases of MOE and hence is a more sensitive investigation. Since the test is based on osteoblast binding, which is also seen in malignancies and trauma, it is not specific to MOE. A gallium 67 scan is positive in soft tissue and bone infections due to its binding to lactoferrin. The uptake becomes negative, once the infection is over. Hence, a gallium 67 scan is far more useful than technetium-99m in monitoring the prognosis and in detection of recurrence. ${ }^{7}$ None of the patients underwent radionuclide scan because it was unavailable in our institution.

Treatment of MOE is mostly medical. Strict glycaemic control was very important in controlling the disease progression. Oral ciprofloxacin was the drug of choice in the past. ${ }^{12}$ However the number of cases showing ciprofloxacin resistance are on an increase due to its irrational unscientific use. Parenteral antibiotics were administered depending on the culture and sensitivity results. Antipseudomonal penicillin like carbenicillin and cephalosporins like ceftazidime and amino glycosides were the most commonly used drugs in this study. However, nephrotoxicity and ototoxicity have to be monitored on administration of aminoglycosides as diabetics are at risk of compromised renal function. Oral fluconazole was given for fungal cases. ${ }^{13}$ The treatment duration was longer in fungal malignant otitis externa. The response to treatment was determined by the improvement in symptoms, decrease in ESR and CRP and disease resolution in gallium 67 scan. Average duration of hospital stay in this study was around 1 month.

Surgery was limited to biopsy and removal of granulation tissue in most patients. However, one patient who had facial palsy due to facial canal involvement underwent facial nerve decompression. There was no improvement after surgery. One patient underwent cortical mastoidectomy as she had chronic otitis media in addition. But there was no pain relief even after the surgery. However, in a study by Jaskaran Singh et al, ${ }^{14}$ resolution of nocturnal pain, hearing loss, ear discharge, granulation and temporomandibular joint pain were better if surgical debridement was done in patients having refractory malignant otitis externa unresponsive to medical treatment.

ESR and CRP were found to elevated in most cases at presentation. It decreased on treatment and hence may be used as a prognostic marker. In this study, every patients were classified into 5 groups based on ESR at presentation and on follow-up. There was a significant fall in ESR and CRP at followup after one month on comparison using paired T test and Wilcoxon signed rank test respectively. Hence, they can be considered as prognostic markers. ${ }^{10}$ On evaluation of patient pain using Wong Baker faces scale and on evaluation of inflammation of ear canal, there was satisfactory response in $43.48 \%$ of the patients, $17.39 \%$ had moderate response and $10.86 \%$ had no response. $28.26 \%$ of the patients died due to other comorbidities before follow up. 5 patients showed complete recovery of facial nerve palsy from grade III to grade I. 7 patients showed improvement in facial nerve palsy from grade III to grade II and one patient showed recovery of facial nerve palsy from grade IV to grade II. One patient showed recovery of glossopharyngeal and vagal nerve palsy. Another patient showed complete recovery of hypoglossal nerve palsy.

\section{CONCLUSIONS}

Malignant otitis externa presents with characteristic nocturnal otalgia. Elderly males were most commonly affected and all patients had type II diabetes mellitus. Bilateral MOE was very rare. Pseudomonas aeruginosa was the most common organism isolated in culture in this study. Fungal infection also caused MOE; the most common organism being Aspergillus and Candida. Glycaemic control with culture directed antibiotics and regular aural toileting for an average duration of one month gave a satisfactory outcome in most cases. ESR and CRP showed reduction on treatment and may be used as a prognostic markers.

Data sharing statement provided by the authors is available with the full text of this article at jemds.com.

Financial or other competing interests: None.

Disclosure forms provided by the authors are available with the full text of this article at jemds.com.

\section{REFERENCES}

[1] Meltzer PE, Kelemen G. Pyocutaneous osteomyelitis of the temporal bone, mandible zygoma. Laryngoscope 1959;69(10):1300-16.

[2] Chandler JR. Malignant external otitis. Laryngoscope 1968;78(8):1257-94.

[3] Franco-Vidal V, Blanchet H, Bebear C, et al. Necrotizing external otitis: a report of 46 cases. Otol Neurotol 2007;28(6):771-3.

[4] Thakar A, Kacker SK, Bahadur S. Malignant external otitis. Indian Journal of Otolaryngology and Head and Neck Surgery 1996;48(2):114-20.

[5] Bhat V, Aziz A, Bhandary SK, et al. Malignant external otitis- a retrospective study of 15 patients treated in a tertiary care center. Journal of International Advanced Otology 2015;11(1):72-6.

[6] Hasibi M, Ashtiani MK, Zarandi MM, et al. A treatment protocol for management of bacterial and fungal malignant external otitis: a large cohort in Tehran, Iran. Ann Otol Rhinol Laryngol 2017;126(7):561-7.

[7] Carfrae MJ, Kesser BW. Malignant otitis externa Otolaryngol Clin North Am 2008;41(3):537-49.

[8] Rajput MSA, Ali-Arain A, Awan MS, et al. Malignant otitis externa: cranial nerve palsies, skull-base erosions and prognosis. Journal of Cranio-Maxillary Diseases 2013;2(2):130-7.

[9] Corey JP, Levandowski RA, Panwalker AP. Prognostic implications of therapy for necrotizing external otitis. Am J Otol 1985;6(4):353-8.

[10] Mani N, Sudhoff H, Rajagopal S, et al. Cranial nerve involvementin malignant external otitis: implications for clinical outcome. Laryngoscope 2007;117(5):907-10. 
[11] Kountakis SE, Kemper JV, Chang CY, et al. Osteomyelitis of the base of the skull secondary to Aspergillus. Am J Otolaryngol 1997;18(1):19-22.

[12] Levenson MJ, Parisier SC, Dolitsky J, et al. Ciprofloxacin: drug of choice in the treatment of malignant external otitis. Laryngoscope 1991;101(8):821-4.
[13] Singh A, Al Khabori M, Hyder MJ. Skull base osteomyelitis: diagnostic and therapeutic challenges in atypical presentation. Otolaryngol Head Neck Surg 2005;133(1):121-5.

[14] Singh J, Bhardwaj B. The role of surgical debridement in case of refractory malignant otitis externa. Indian J Otolaryngol Head Neck Surg 2018;70(4):549-54. 